\title{
Etica della responsabilità in nefrologia
}

\author{
Mario Timio ${ }^{1,2}$, Francesca Timio ${ }^{1}$ \\ ${ }^{1}$ Dipartimento di Medicina Interna e Nefrologia e Dialisi, Ospedale di Foligno, Foligno (PG) \\ ${ }^{2}$ Direzione Scientifica Centro di Bioetica, Regione Umbria, Perugia
}

\begin{abstract}
Responsability ethics in nephrology
Responsability bioethics includes informed consent and timely appropriate information. In nephrology informed consent, coined by the Italian lawyer Filippo Grispigni, becomes the central focus of many diagnostic procedures and therapeutic approaches including dialysis. The information related to every clinical care and to end of life must be achieved by good communication and timely discussion between the health care team, the patient and the patient's family. Thus according to bioethicist Authorities while dialysis may be technically possible for the patients with multiple co-morbidities and diminishing quality of life it may not be the kind of care they would choose if they had full understanding of their prognosis. Interviewed patients with ESRD agreed that facilitated advance care planning through the provision of timely appropriate information can positively enhance rather than diminish their hope. This is the real goal of modern bioethics.
\end{abstract}

Keywords: Informed consent, Responsibility, Timely information

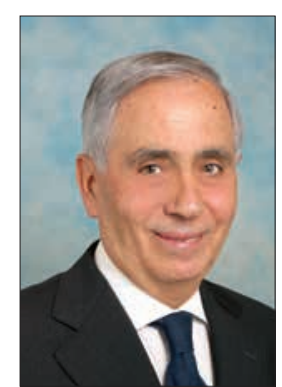

Mario Timio

\section{Concetto di autodeterminazione nelle scelte cliniche}

La bioetica, come qualunque altro ramo dell'etica, si fonda sul principio di responsabilità e tale principio non può che, logicamente, affondare le proprie radici sul principio di autodeterminazione. Infatti ogni teoria etica riconosce che l'uomo è soggetto e oggetto di diritti e doveri. Ma tale attribuzione di diritti e doveri all'uomo trova la propria giustificazione nell'idea che tale soggetto sia responsabile delle proprie azioni, che, cioè, "sia consapevole delle conseguenze derivanti della propria condotta" e che sia in grado appunto di $<$ responsare > o <rispondere> (1). È da chiarire allora su cosa si fonda il concetto di responsabilità. Sul piano strettamente logico essere responsabili delle proprie azioni implica che ciascuna persona sia libera di operare delle scelte, ossia sia capace di autodeterminazione. Quale senso avrebbe conside-

Accepted: January 15, 2017

Published online: February 13, 2017

Indirizzo per la corrispondenza:

Prof. Mario Timio

Via XX Settembre 22

06121 Perugia

timma@libero.it rare un nefropatico responsabile per qualcosa che ha fatto o non fatto perché costretto? Per esempio, scegliere la dialisi peritoneale o l'emodialisi come mezzo di cura dell'insufficienza renale cronica? Per restare in questo campo vediamone un'applicazione con la quale i medici quotidianamente sono impegnati: I'istituto del <consenso informato>. Proprio per questo quotidiano impegno forse ai medici sfugge l'origine di tale istituto. Ma non solo i medici, ma anche i giuristi e i filosofi non polarizzano adeguatamente l'attenzione sulla genesi del <consenso informato>. Ha origini lontane, anche perché si radica nella dottrina della cosiddetta "scuola positiva". Non a caso chi per primo ne parla è un penalista italiano nato nel 1884 , tal Filippo Grispigni che in saggi che risalgono al 1914 e oltre, con mente filosofica e analitica, fissa alcuni punti ancor oggi validi. Egli scrive: "Un trattamento medico-chirurgico, compiuto bensì secondo le regole dell'arte medica, ma senza il valido consenso del paziente o del suo rappresentante legale, costituisce, a meno che non si verta in stato di necessità, un fatto civilmente illecito. E, dal punto di vista penale, se l'esito dell'operazione è sfavorevole, costituisce il delitto di lesione personale o di omicidio colposo; mentre, se l'esito è favorevole, può dare luogo ... a un delitto contro la libertà" (2). Certo che le parole di Grispigni sono molto dure per i medici e tutte a favore dei pazienti. Ma il focus a lui caro, che si deduce anche dalle ultime parole, è proprio questo: un valido consenso è condizione necessaria al trattamento medico-chirurgico se non si vuole violare le libertà di scelta del paziente. Ed è questa idea del rispetto della libertà del paziente la base su cui giace l'attuale "consenso informato", come anche il "consenso valido". Allora se la responsabilità e l'autodeter- 
minazione sono la base della bioetica, è logico sostenere che entrambe legittimino il rispetto del principio di uguaglianza. Un principio che scaturisce ancora dal citato Grispigni. Infatti il riconoscimento del valore della libertà di scelta agisce in due diverse ma complementari e inseparabili direzioni. Da un lato c'è il <diritto> al rispetto della propria libertà di scelta e, dall'altro, il < dovere> e il rispetto dell'altrui libertà di scelta. È come dire che tu medico non puoi agire contro il mio consenso o dissenso, ma anche io paziente non debbo contravvenire al tuo consenso o dissenso che sia. Questo pone, proprio per effetto del pensiero di Grispigni, il principio di reciprocità e di uguaglianza. Un concetto che sta riemergendo anche nella nostra Magistratura sull'annoso quotidiano contrasto tra medici e pazienti.

\section{La bioetica come collante tra libertà e uguaglianza}

Non di rado, libertà e uguaglianza sono stati considerati valori in conflitto. A margine di un recente Congresso di Bioetica, la giurista Simona Sagnotti ha sostenuto che proprio nella Bioetica si può trovare quel collante che rende tale contrapposizione sanabile o almeno non alternativa. O meglio l'alternativa libertà/uguaglianza non è nello stato delle cose ma nel modo in cui lo approcciamo. Allora la mia libertà non trova un ostacolo nella libertà altrui, ma in essa incontra il giusto limite. "Giusto perché rispettoso del principio di uguaglianza. La mia liberà vale la tua. Di più: vale perché vale la tua. Così la libertà dell'altro diviene veicolo di valore per la propria, in un rapporto di reciproco rispetto" (3). In questo contesto si erge la Bioetica della responsabilità, come ulteriore contributo alla scoperta dell'uomo che soffre e di un altro uomo che tenta di lenire il suo dolore. Che è poi la Bioetica della parità tra due. Facciamo allora scendere la Bioetica in campo nefrologico cercando di cogliere quanto la disciplina possa contribuire a migliorare l'istituto del Consenso Informato basato sul principio del rispetto dell'altro. Intanto parliamo di informazione, che è la base di ogni consenso diagnostico e terapeutico, ma anche di pratiche che si attivano durante un trattamento o di spiegazioni che il paziente chiede o che si danno in previsione di particolari eventi, compresa la morte. Ciò significa che è il medico che, usando il massimo grado di correttezza possibile, determina il contenuto della propria prestazione, anche perché, almeno di regola, il paziente non possiede adeguate conoscenze tecniche (e quando mostra di possederle attingendo a notizie spot dai mass media riesce talvolta a incrinare un corretto rapporto medico-malato) e si affida fiduciariamente a lui. Ma, per il nefrologo che ha in cura un dializzato, tale fiducia, proprio per la continuità del rapporto, deve essere rafforzata ad ogni procedura diagnostica e terapeutica. In che modo? Da una parte puntando sull'autonomia del nefrologo - che poi significa responsabilità - di scelte professionali basate su evidenze scientifiche e sperimentali, dall'altra sulla puntuale comunicazione con il paziente informato sulle motivazioni delle scelte funzionali alla sua salute (4).
Alcune esemplificazioni. Se occorre revisionare la fistola A.V. occore spiegare, per ottenere il consenso condiviso, che l'anomalo funzionamento dell'accesso vascolare, che rende inefficace la tecnica emodialitica, è nocivo per la salute del paziente. È questo tipo di comunicazione che crea e rafforza empatia tra medico e nefropatico. Parimenti il consenso condiviso può emergere quando il medico si fa carico di illustrare i vantaggi cardiovascolari, e non solo, della modifica di un programma diagnostico e terapeutico volto a controllare il rapporto $\mathrm{Ca} / \mathrm{P}$ e i segni di iperparatiroidismo che può richiedere anche l'ablazione ghiandolare. Allora di nuovo tecnica comunicativa qualificata per avere qualificati approcci clinici. Ancora comunicazione che sarà parimenti efficace quando si prospetta la necessaria coronarografia (o tecniche diagnostiche equivalenti) a un dializzato per essere inserito nella lista attesa di trapianto renale. Quindi reiterata comunicazione nefrologo-nefropatico base di un corretto e responsabile approccio alla pratica medica. Con un sottolineatura in più. II nefrologo è l'unico medico nell'universo ospedaliero che costruisce quotidianamente, a lungo e con il medesimo paziente un approccio saldo e globale con la comunicazione franca, esaustivo e qualificata. Tutto ciò come epifenomeno della bioetica della responsabilità.

\section{La chiave bioetica sul fine vita dei dializzati}

In una chiara relazione sul fine vita dei dializzati esposta ad Assisi, la bioeticista Elisabeth Brown dell'Hammersmith Hospital di Londra fuga ogni paura di avere un colloquio franco con i pazienti circa l'outcome della pratica dialitica. Un colloquio che non distrugge speranze né pone problemi sulla conoscenza anticipata della ridotta aspettativa di vita (5). Alla stessa conclusione erano giunti Davison e Simpson i quali, intervistando dei pazienti con ESRD, avevano tratto certezze circa il fatto che l'appropriata e precoce informazione sul "destino" dei nefropatici rafforza, e non indebolisce, la loro speranza nella vita e la fiducia nel medico (6). Sempre dall'UK fanno sapere che dall'informazione traggono vantaggio anche i familiari i quali messi per tempo al corrente della prognosi del loro congiunto sono preparati a qualsiasi evento avverso (7). Ma questo aspetto gli italiani lo conoscono da tempo senza forse disporre di adeguate conoscenze bioetiche. Quello che invece gli inglesi insegnano è il periodo "ideale" per discettare di prognosi della malattia e di fine vita. Ci sono specifiche fasi dell'iter della malattia di cui discutere che fanno parte di una buona pratica clinica e che conducono il paziente a un cambiamento atraumatico delle proprie abitudini cliniche e a un'appropriata attenzione nel controllo dei sintomi e delle componenti emotive e psicosociali legate o meno alla dialisi. I tempi giusti per parlare del decorso della malattia e di fine vita sono riassunti nella Tabella I. 
TABELLA I - Tempistica comunicativa

$$
\begin{aligned}
& \text { - Quando si programma la dialisi se il paziente ha scelto la terapia } \\
& \text { conservativa } \\
& \text { - Quando il paziente peggiora malgrado il trattamento dialitico } \\
& \text { - Dopo un serio evento clinico (stroke, diagnosi di cancro, frattura } \\
& \text { del femore) } \\
& \text { - Quando sopravviene un'insufficienza renale complicante serie } \\
& \text { malattie (cancro, scompenso cardiaco) } \\
& \text { - Il paziente non vuole ritornare in dialisi dopo l'insuccesso del } \\
& \text { trapianto } \\
& \text { - Il paziente non vuole iniziare l'emodialisi dopo l'insuccesso della } \\
& \text { dialisi peritoneale e l'indisponibilità di un trapianto } \\
& \text { - Seri problemi di accessi vascolari del paziente che rifiuta la di- } \\
& \text { alisi peritoneale e il trapianto non è disponibile } \\
& \text { - Il paziente desidera sospendere la dialisi }
\end{aligned}
$$

\section{Indicazione sul timing della comunicazione}

Indicazioni bioetiche sul timing nel trattare argomenti relativi alla prognosi della malattia e di fine vita provengono ancora dall'UK in termini di Linee Guida. Tutti i pazienti con malattie croniche, compresa la ESRD, sono avviati a colloquio precoce con il team curativo sull'evoluzione della malattia e sul destino finale, senza "sentimentalismi" né falsi messaggi consolatori (8). Il processo è denominato advance care planning. Certo, alcuni nefrologi non hanno tempo disponibile per attivare tale processo, oberati come sono da altre mansioni ritenute più utili per l'outcome del paziente. Ma questi identifica la paura del dolore, la perdita di dignità, l'abbandono e la solitudine nel silenzio del medico. Eppure al medico sono richiesti, anche in questo contesto, onestà e approccio diretto, sensibilità nell'annunciare brutte notizie, ascolto attivo del paziente e incoraggiamento e discrezionalità nel parlare di morte e del morire, ma serve anche la predisposizione del paziente a essere guidato dal medico. Anche se per alcuni nefrologi queste pratiche sono quotidiane, per altri è difficile attuarle, non perché non le conoscono (non è più necessario andare nel Regno Unito per apprenderle e realizzarle), ma perché le ritengono time consuming in un contesto clinico e assistenziale dove si ha sempre poco tempo. Allora il discorso si sposta dal contesto bioetico a quello organizzativo e quindi politico. Ma questo è un altro ambito che esula dagli obiettivi di questa rubrica.

\section{Conclusioni}

Nell'etica della responsabilità possono essere inseriti il consenso informato e la comunicazione esplicativa all'insegna della scelta libera del paziente. II riconoscimento del valore della libertà di scelta agisce in due complementari direzioni: il "diritto" al rispetto della propria libertà di scelta e il "dovere" al rispetto dell'altrui libertà di scelta. Ciò significa, per tradurre questo assunto bioetico nella pratica quotidiana della nefrologia, che ci siano rispetto e accettazione della scelta del paziente ma anche del medico. Se manca questa simmetria cade ogni presupposto di corretta e reciproca accettazione nel menage quotidiano assistenziale. La comunicazione esplicativa come segmento della bioetica clinica si basa sulla corretta, onesta, chiara e tempestiva comunicazione dell'outcome nefrologico nella fase predialitica e dialitica, compresa la dura problematica della morte e del modo di affrontarla. Viene riportata la pratica illuminante proveniente dal Regno Unito, ove l'accesso precoce a franche pratiche bioetiche comunicative conduce a mitigare lo stress della pratica dialitica e alla gestione "serena" del trapasso.

\section{Disclosures}

Financial support: No financial support was received for this submission. Conflict of interest: The authors have no conflict of interest.

\section{Bibliografia}

1. Sagnotti SC. La bioetica della responsabilità, in AAVV. Le Giornate della Bioetica. Università degli Studi di Perugia. 2010:208.

2. Grispigni F. La responsabilità per il trattamento medico-chirurgico <arbitrario>, in "La Scuola Positiva" 1914;5:24-29.

3. Sagnotti SC: op.cit. n.1.

4. Sassi A. Profili giuridici del Consenso Informato al trattamento terapeutico, in AAVV. Seminario di Bioetica. Aracne Editore, Roma. 2008:441.

5. Brown E. End of life discussion in nephrology. In: Timio M. et al. Cardionephrology 11. Nuova Bios Editore, Castrolibero (CS) 2010:21.

6. Davison SN, Simpson C. Hope and advance care planning in patients with end stage renal disease: quantitative interview Study. Brit Med J. 2006;333:886-891.

7. Apatira L, Boyd EA, Malvar G, et al. Hope, truth and preparing for death: perspectives of surrogate decision makers. Ann Int Med. 2008;149:861-868.

8. Royal College of General Practitioners, British Geriatric Society, Royal College of Physicians, et al. Advance Care Planning: National Guideline. Royal College of Physicians. London 2009. 